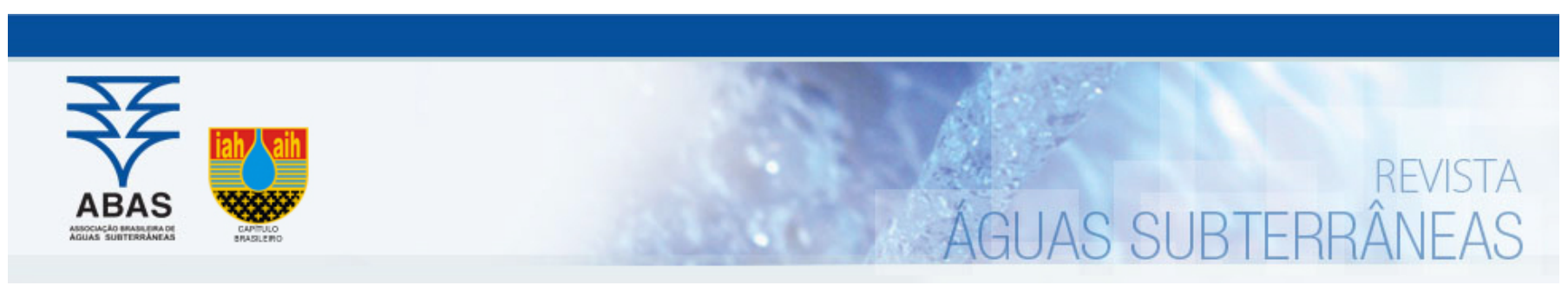

Artigos

\title{
Avaliação numérica da eficiência da remediação de plumas de nitrato a partir de pulsos de injeção de etanol
}

\section{Reactive transport model to evaluate the nitrate remediation effectiveness based on ethanol injection pulses}

\author{
Elias Hideo Teramoto1,2; Hung Kiang Chang1,2,3凶 \\ ${ }^{1}$ Lebac - Laboratório de Estudos de Bacias, Instituto de Geociências e Ciências Exatas, UNESP - Campus de Rio Claro, SP \\ 2 Centro de Estudos Ambientais (CEA), UNESP - Campus de Rio Claro, SP \\ ${ }^{3}$ Departamento de Geologia Aplicada, Instituto de Geociências e Ciências Exatas, UNESP - Campus de Rio Claro, SP \\ \eh teramoto@yahoo.com.br, chang@rc.unesp.br
}

Resumo

Palavras-chave:

Nitrato.

Contaminação de Águas Subterrâneas.

Remediação.

Transporte Reativo.

Keywords

Nitrate.

Groundwater Contamination.

Remediation.

Reactive Transport Model.

Revisado por pares.

Recebido em: 02/03/2018.

Aprovado em: 04/06/2018.

\begin{abstract}
O nitrato representa, em termos mundiais, o contaminante mais disseminado em aquíferos rasos, oferecendo riscos à saúde humana pela ingestão de água subterrânea contaminada. Deste modo, a descontaminação in situ do aquífero deve ser considerada. Embora testes de campo sejam escassos, testes laboratoriais têm indicado a forte eficiência do etanol na redução do nitrato. Com vistas a avaliar a viabilidade do emprego de técnicas de remediação baseadas na desnitrificação induzida pela injeção de solução com elevadas concentrações de etanol, foram utilizadas simulações numéricas de transporte reativo em área contaminada do Aquífero Rio Claro. Os resultados apontam que, nas condições técnicas impostas, a remediação foi eficiente para remoção do nitrato, restituindo a potabilidade da água subterrânea por um período superior a 500 dias. Contudo, para que essa solução seja eficiente em longo prazo deve ser combinada com a eliminação da fonte de contaminação, para evitar o aporte de novos contaminantes.
\end{abstract}

\section{Abstract}

Nitrate represents the most widespread contaminant in shallow aquifers, and offers risk to the human health through ingestion of contaminated groundwater. Thus, in situ aquifer treatment is highly recommended. Laboratory tests indicated the strong ethanol efficiency in nitrate reduction, but field test are scarce. Numerical simulations of reactive transport were carried out in order to evaluate the feasibility of remediation techniques based on denitrification induced by solution injection with high concentrations of ethanol in the Rio Claro Aquifer, located in the homonymous municipality. The results point out that the tested remediation, under the imposed technical conditions, it was efficient for nitrate removal and is capable of restoring the groundwater potability. However, for long term efficiency it is necessary to combine the proposed solution with source of contamination removal to prevent the input of new contamination inlet into the aquifer.

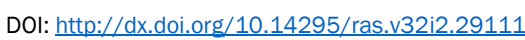

\section{INTRODUÇÃO}

O risco para a saúde humana ao consumir água com concentrações elevadas de nitrato é decorrente da transformação deste em nitrito no sistema digestivo. O nitrito oxida o ferro na hemoglobina dos glóbulos vermelhos para formar metahemoglobina, que não possui a mesma capacidade de transporte de oxigênio da hemoglobina (WEITZBERG \& LUNDBERG, 2013). Isso cria a condição conhecida como metahemoglobinemia (às vezes referida como "síndrome do bebê azul"), na qual o sangue não tem a capacidade de transportar oxigênio suficiente para as células do corpo individuais, fazendo com que as veias e a pele se tornem azuis.
O vazamento de esgoto em ambientes urbanos (ZHANG et al., 2015; GRIMMEISEN et al., 2017; VYSTAVNA et al., 2017) e o uso de fertilizantes (LOCKHART, KING \& HARTER, 2013; EXNER et al., 2014; WANG et al., 2016) representam as principais fontes de contaminação por nitrato na água subterrânea. No território brasileiro são abundantes os estudos de caso descrevendo a contaminação por nitrato na água subterrânea destinada ao abastecimento público e privado, como por exemplo, em Castro et al. (1992), Melo et al. (1998) e Montanheiro (2014).

Em ambientes oxidantes, o nitrato é estável e a desnitrificação, isto é, a redução do $\mathrm{NO}_{3}$ para $\mathrm{N}_{2}$, é pouco expressiva e as plumas 
de nitrato se prolongam por grandes distâncias. A remediação in situ do nitrato consiste em adicionar uma espécie química na forma reduzida, favorecendo uma reação de oxirredução, na qual o nitrato é reduzido e a espécie reduzida adicionada é oxidada. Robertson et al. (2000), Schipper \& Vojvodic-Vukovic (2000) e Su et al. (2007) demonstraram a viabilidade do emprego de barreiras reativas de matéria orgânica vegetal para promover a remediação do nitrato. A injeção de substâncias inorgânicas na forma reduzida, como a pirita (TORRENTÓ et al., 2011; PU et al., 2014), o Fe(II) (TAl et al., 2009) e o ferro zero-valente (FU et al., 2014), mostraram-se igualmente eficientes para promover a redução do nitrato em testes de laboratório.

Compostos orgânicos solúveis em água, incluindo acetato (MARTIN et al., 2009), sacarose (GOMEZ et al., 2000), metanol (MOHSENIBAND \& ELLIOTT, 1998, GOMEZ et al., 2000) e metanoato (SMITH et al., 2001), podem eficientemente ser empregados como fonte de carbono para promover a redução autotrófica e heterotrófica do nitrato. Dentre os compostos orgânicos para promover a redução do nitrato, o etanol têm merecido destaque por sua eficiência (MOHSENI- BANDPI \& ELLIOTT, 1998; GOMEZ et al., 2000; MARTIN et al., 2009; SALMINEN et al., 2014). Na comparação com os demais compostos orgânicos, o etanol é mais eficiente na redução do nitrato que o acetato (MARTIN et al., 2009), metanol (GOMEZ et al., 2000; MOHSENI-BANDPI \& ELLIOTT, 1998), ácido acético (MOHSENI-BAND \& ELLIOTT, 1998) e sacarose (GOMEZ et al., 2000).

A eficiência do etanol na redução do nitrato está associada à sua facilidade de biodegradação em condições naturais. Os valores de cinética de decaimento de primeira ordem média, determinada por Zhang et al. (2006), em condições de campo, é de 0,32 dias-1, o que corresponde a um tempo de meia-vida de 2,2 dias. Acrescenta-se ainda que, por ser completamente solúvel em água, sua dispersão no aquífero é favorecida, além de possuir baixo custo relativo.

Diversas alternativas para tratamento de águas subterrâneas contaminadas por nitrato foram demonstradas com sucesso em incontáveis experimentos laboratoriais ou em escala reduzida. Por outro lado, trabalhos envolvendo a remediação in situ de águas subterrâneas contaminadas por nitrato são bastante escassos, existindo fortes incertezas quanto à eficiência das ações mitigadoras deste cenário de contaminação. Com o intuito de avaliar conceitualmente se a injeção de etanol pode ser considerada uma alternativa viável de remediação de aquíferos contaminados por nitrato, o presente trabalho avalia, por intermédio de simulações numéricas de transporte reativo, a eficiência desta alternativa de remediação.

\section{MATERIAIS E MÉTODOS}

\section{1. Área de estudo}

Para realizar as simulações pretendidas no presente estudo, foi selecionada uma área onde a contaminação por nitrato tenha sido reconhecida e existam dados relativos à caracterização hidrogeológica, permitindo a devida parametrização dos modelos a serem empregados.

A área de estudo para a realização das simulações foi o Campus da UNESP de Rio Claro (Figura 1), onde anteriormente foi conduzida a caracterização hidrogeológica conduzida por diversos trabaIhos, incluindo Oliva et al. (2005), Oliva (2006), Neto et al. (2016) e Gonçalves \& Chang (2018). Na área do campus, o Aquífero Rio Claro é predominantemente arenoso e com elevados valores de condutividade hidráulica, mas em algumas porções do aquífero existem lentes argilosas relativamente espessas, como indicam as descrições de sondagens realizadas na área (Figura 1b). A espessura do Aquífero Rio Claro, determinada por sondagens, varia entre 14,4 m e 21,0 m; o fluxo na região de interesse é NE-SW e possui um gradiente hidráulico médio de 0,0075.

Grande parte do Aquífero Rio Claro encontra-se sob a área urbana do município de Rio Claro/SP, o que o torna fortemente susceptível à contaminação por vazamentos da rede de esgoto ou fossas sépticas. Toda a água que abastece o Campus da UNESP de Rio Claro é subsuperficial e a maior parte provém do Aquífero Rio Claro. Um poço de bombeamento está interditado desde junho de 2014 (PM-08), período em que foram determinadas concentrações de NO3- de 48,8 mg/L, o que equivale a $11 \mathrm{mg} / \mathrm{L}$ de N-NO3, superior ao limite de potabilidade de $10 \mathrm{mg} / \mathrm{L}$. 
Figura 1 - a) Área de estudo, com a distribuição dos poços de monitoramento e poços de bombeamento, bem como o mapa potenciométrico. b) Perfil geológico do Aquífero Rio Claro, determinado a partir de um ponto de sondagem (SP-04) na área
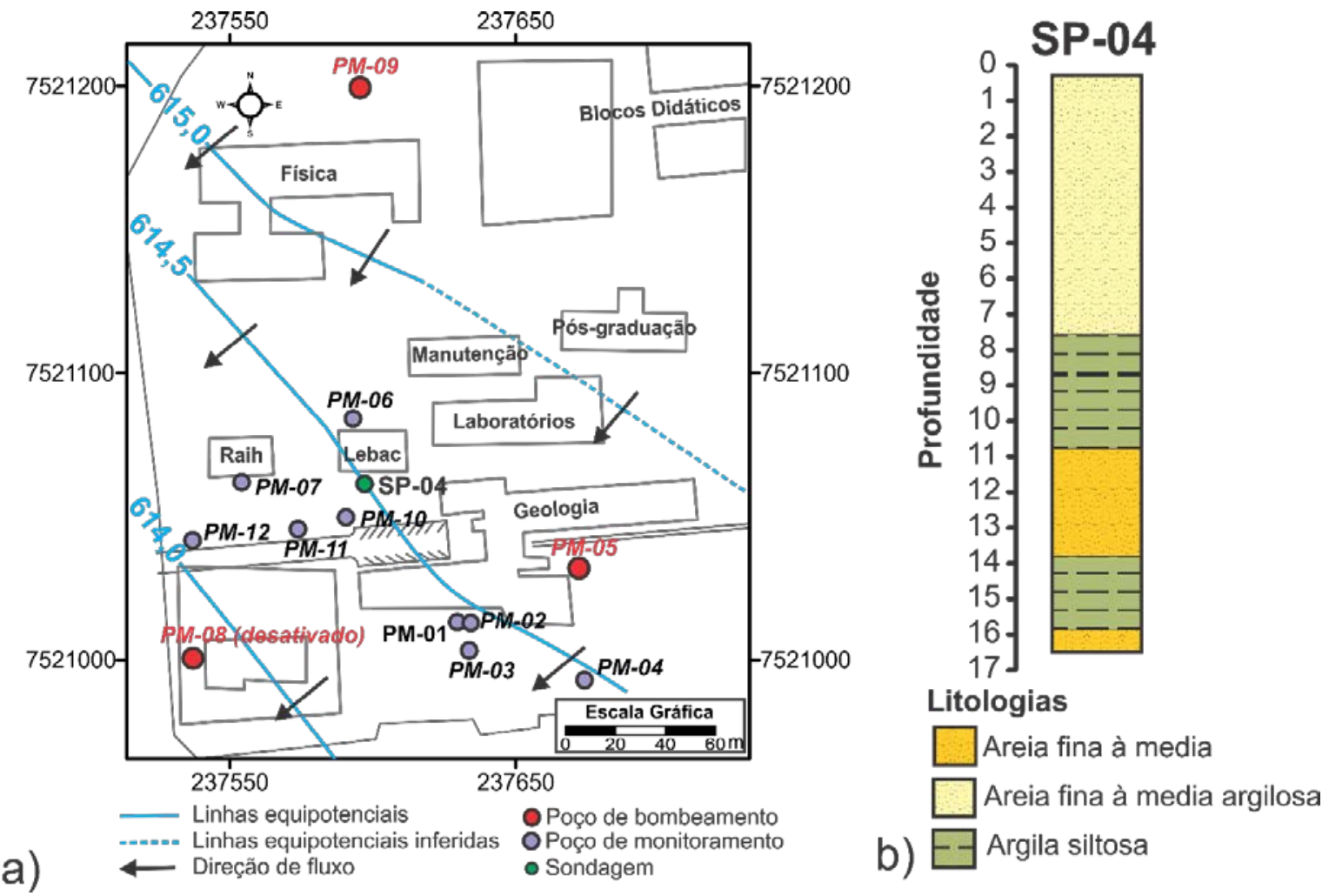

\subsection{Modelo conceitual}

Os resultados dos trabalhos de Macmahon et al. (1998) e Zhang et al. (2006), que descreveram experimentos com a injeção de etanol em aquíferos rasos, serviram como referências para a ela- boração do modelo conceitual adotado no presente trabalho. Entretanto, ao contrário desses trabalhos, o método de injeção idealizado foi por direct-push, a partir de um único pulso de injeção de solução contendo etanol (Figura 2), e não a partir de poços, com períodos prolongados de injeção.

Figura 2 - Ilustração esquemática da injeção de etanol por equipamento baseado no método direct-push

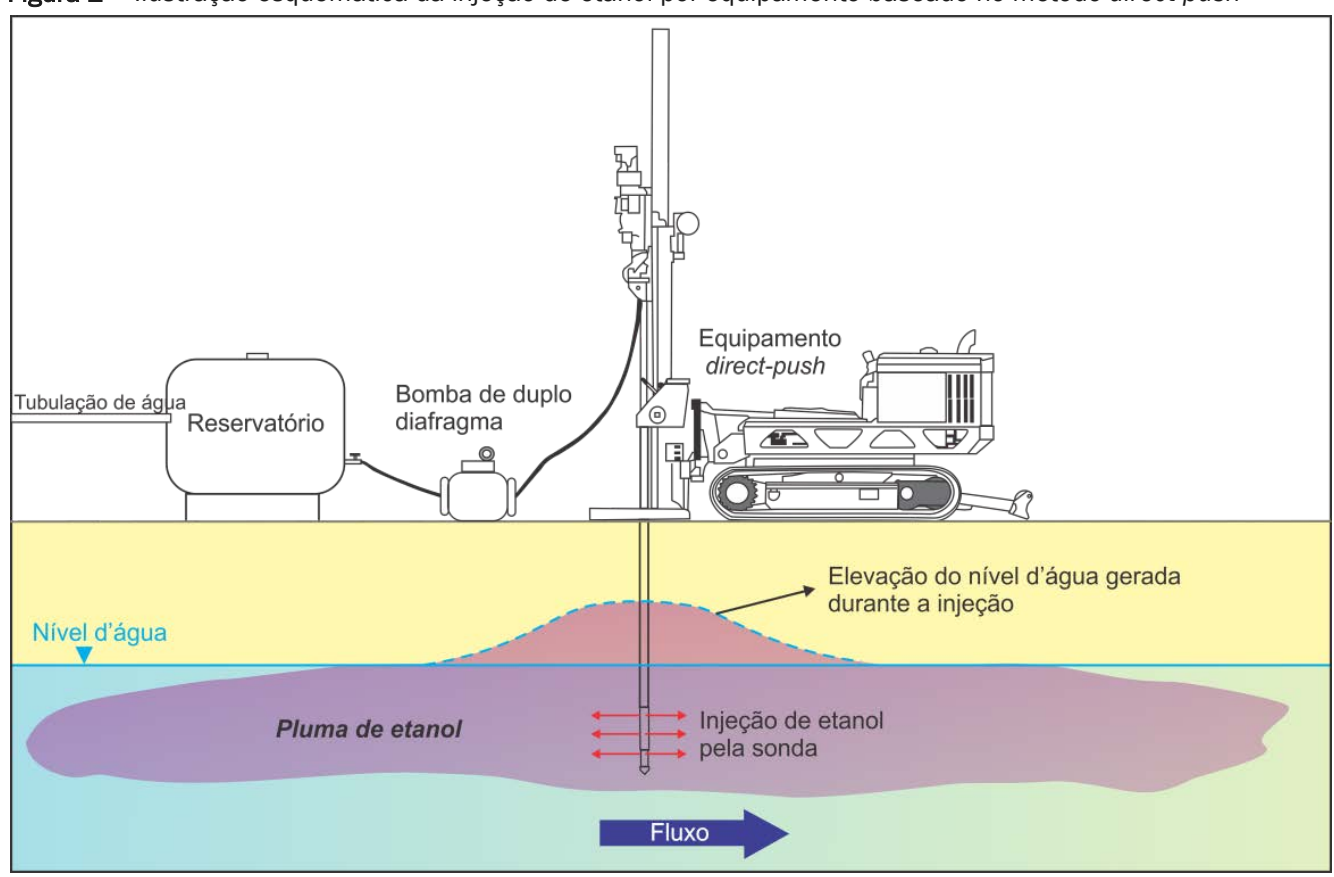


Em razão da disponibilidade de oxigênio no aquífero, inicialmente o etanol é degradado por biodegradação aeróbica (Equação 1), tendo em vista que esta representa a reação termodinamicamente mais favorável.

$$
3 \mathrm{O}_{2}+\mathrm{C}_{2} \mathrm{H}_{5} \mathrm{OH} \rightarrow 3 \mathrm{H}_{2} \mathrm{O}+2 \mathrm{CO}_{2}
$$

A taxa constante de reação da Equação 1 ( $\left(\right.$ axa $\left.a_{a e r}\right)$ segue provavelmente um modelo cinético de segunda ordem (Equação 2), uma vez que está relacionado à disponibilidade de $\mathrm{O}_{2}$ e etanol na água e é proporcional à constante de taxa de reação $\left(k_{\text {aer }}\right)$.

$$
\text { taxa }_{\text {aer }}=k_{\text {aer }}[\text { Etanol }]\left[\mathrm{O}_{2}\right]
$$

A redução do nitrato por oxidação do etanol (Equação 3) ocorre apenas quando todo o oxigênio é consumido e o meio se torna anóxico, uma vez que a desnitrificação é inibida na presença de oxigênio dissolvido na água (MACMAHON et al., 1998).

$$
12 \mathrm{NO}_{3}^{-}+5 \mathrm{C}_{2} \mathrm{H}_{5} \mathrm{OH} \rightarrow 6 \mathrm{~N}_{2}+9 \mathrm{H}_{2} \mathrm{O}+10 \mathrm{CO}_{2}+12 \mathrm{OH}^{-}
$$

Tendo em vista que a taxa de degradação do etanol via redução do nitrato é intrinsicamente dependente da atividade do etanol e do próprio nitrato na água, esta reação segue um modelo cinético de segunda ordem (Equação 4). Desta maneira, a taxa de biodegradação do etanol pela redução do nitrato $\left(\operatorname{tax} a_{\text {rednit }}\right)$ é igual a constante de taxa de reação da degradação do etanol via redução do nitrato $\left(k_{\text {rednit }}\right)$ multiplicado pela atividade do etanol e nitrato na água. Para representar a inibição da redução do nitrato pela presença do oxigênio, foi acrescida a atividade do $\mathrm{O}_{2} \mathrm{com}$ expoente negativo, indicando que a taxa de desnitrificação cresce à medida que a concentração de $\mathrm{O}_{2}$ decresce.

$$
\text { taxa } a_{\text {rednit }}=k_{\text {rednit }}[\text { Etanol }]\left[\mathrm{NO}_{3}^{-}\right]\left[\mathrm{O}_{2}\right]^{-1}
$$

Caso todo $\mathrm{o}_{2}$ e o nitrato tenham sido consumidos, a biodegradação do etanol ocorrerá via redução do Fe(III) presente no sedimento, redução do $\mathrm{SO}_{4}{ }^{2-}$ e por fim, pela metanogênese.

\subsection{Simulação numérica de transporte reativo}

As simulações de transporte reativo foram realizadas empregando o aplicativo x2t, que compõe o Geochemist's Workbench (BETHKE \& YEAKEL, 2014). O modelo foi representado por um domínio bidimensional com $200 \mathrm{~m}$ de comprimento, $100 \mathrm{~m}$ de largura e 4 $m$ de espessura, discretizado em uma malha de diferenças finitas com 100 linhas e 50 colunas de $2 \mathrm{~m}$ de largura. As condições de contorno empregadas nas bordas leste e oeste foram do primeiro tipo (carga especificada), de modo a reproduzir um gradiente hidráulico de 0,0075, compatível com as observações de campo.

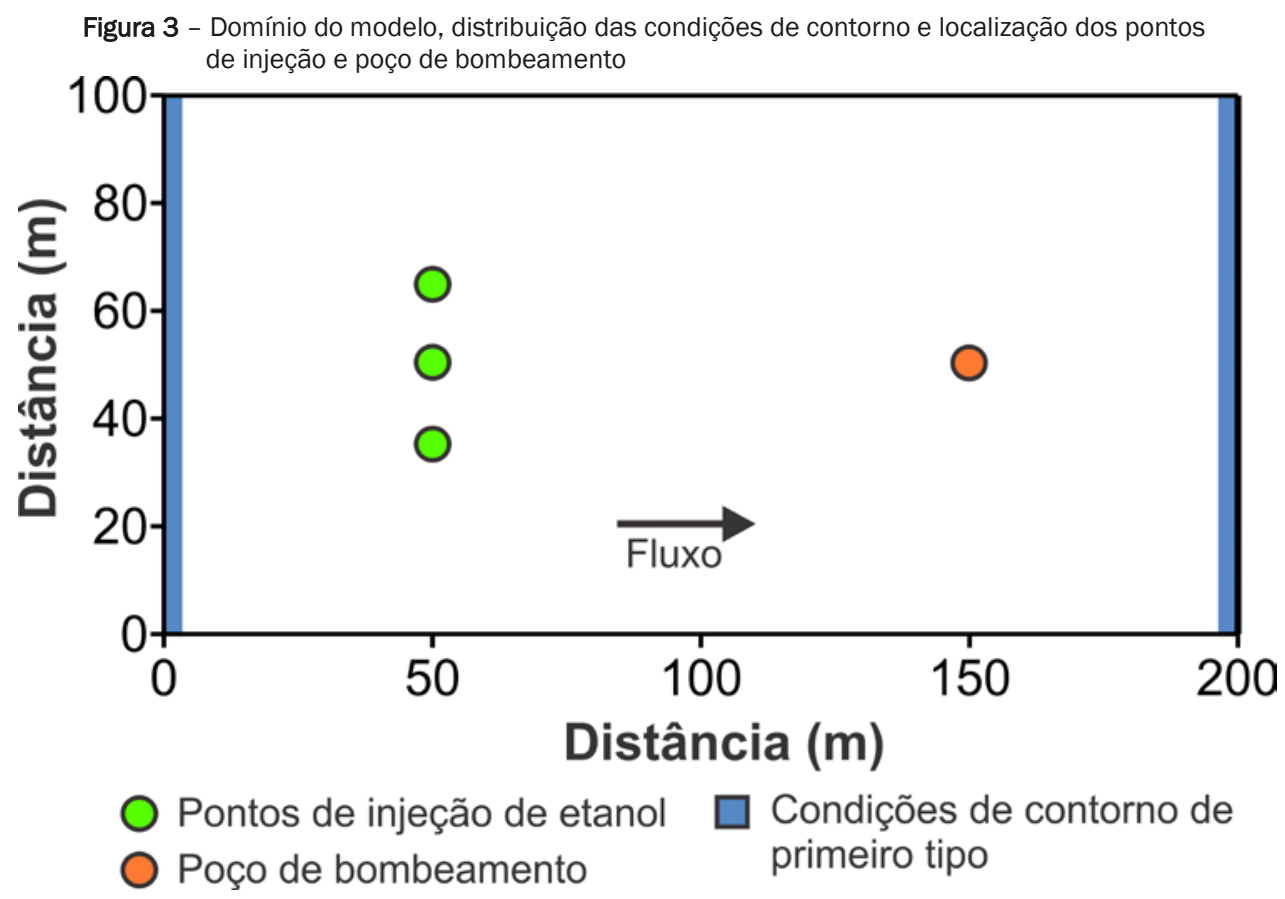

O valor de condutividade hidráulica adotado foi de $3,82 \times 10^{-5}$ $\mathrm{m} / \mathrm{s}$, valor que representa a média geométrica dos três ensaios de slug apresentados por Oliva et al. (2005) no Campus da UNESP; a porosidade efetiva adotada foi 0,17 , com base no trabalho de Neto et al. (2015). Em relação aos parâmetros de transporte foi admitida uma dispersividade longitudinal de 0,6 m e uma dispersividade transversal de 0,001 $\mathrm{m}$. O trabalho experimental de Zhang et al. (2006) verificou que o etanol não sofre retardamento dentro aquífero e, por esta razão, esta condição foi adotada no modelo.

Para simular a biodegradação do etanol foram utilizadas as rea-

ções que seguem os modelos cinéticos descritos nas Equações 2 e 4. 0 coeficiente de taxa de reação empregado para as constantes de taxa de reações de biodegradação por via aeróbica $\left(k_{a e r}\right)$ e por redução do nitrato $\left(k_{\text {rednit }}\right)$ são, respectivamente, 6,0 x 10-4 $\mathrm{mol} / \mathrm{Kg} / \mathrm{s} \mathrm{e} 4,0 \times 10^{-4} \mathrm{~mol} / \mathrm{Kg} / \mathrm{s}$. Os valores das taxas de reação empregadas no modelo se basearam no tempo de meia-vida de 2,2 dias, reportadas no trabalho de Zhang et al. (2006).

Para representar os poços de bombeamento instalados na área de estudo, foi inserido um poço distante $150 \mathrm{~m}$ a jusante da borda oeste do modelo (Figura 3). A vazão deste poço de bombeamento foi de $10 \mathrm{~m}^{3} /$ dia, valor próximo da média dos três poços de bombeamentos presentes no campus. 
Em ambientes urbanos, o nitrato se distribui como grandes e extensas áreas contaminadas. Deste modo, foi adotada a premissa de que todo o aquífero na área está contaminado por uma concentração de $48,8 \mathrm{mg} / \mathrm{L}$ de nitrato na simulação. Tendo em vista que o aquífero é do tipo livre, foi imposta a condição de que o pH está em equilíbrio com uma pressão parcial de $\mathrm{CO}_{2}$ igual a 10-1,5 atm. Tendo em vista que a solubilidade do oxigênio dissolvido é 8,2 $\mathrm{mg} / \mathrm{L}$ a $25^{\circ} \mathrm{C}$, foi imposta esta concentração na composição inicial do aquífero.

A injeção se procedeu em três pontos, situados $100 \mathrm{~m}$ a montante do poço de bombeamento e $50 \mathrm{~m}$ a jusante da borda oeste do mo- delo (Figura 2). Em cada ponto foi injetada uma solução contendo $48,8 \mathrm{~g} / \mathrm{L}$ de etanol, com pressão de injeção de 10 psi por um período de cinco horas, reproduzindo as condições operacionais de campo. A opção pela não injeção de etanol em sua forma pura se dá para prevenir a sua possível distribuição na porção superior da zona saturada na forma de fase líquida não-aquosa, o que limitaria a eficiência da remediação. A Tabela 1 apresenta as composições iniciais da solução do aquífero e que entram no modelo pelo contorno oeste do modelo e a composição da solução injetada. A composição inicial se baseou em análises químicas realizadas em junho de 2014 .

\begin{tabular}{ccc}
$\begin{array}{c}\text { Tabela } 1 \text { - Composição química inicial do aquífero e do fluido injetado empregada } \\
\text { nas simulações realizadas para remediação do nitrato }\end{array}$ \\
\hline Parâmetro & $\begin{array}{c}\text { Composição inicial/ solu- } \\
\text { ção que entra pelo con- } \\
\text { torno oeste }\end{array}$ & Solução injetada \\
\hline \hline pH & $\begin{array}{c}\text { Controlado pelo equilíbrio } \\
\text { com PCO }{ }_{2}=10^{-1,5} \text { atm }\end{array}$ \\
$\mathrm{O}_{2(\mathrm{aq})}(\mathrm{mg} / \mathrm{L})$ & 8,2 & 7,2 \\
$\mathrm{Na}^{+}(\mathrm{mg} / \mathrm{L})$ & 20,7 & $1,0 \times 10^{-3}$ \\
$\mathrm{~K}^{+}(\mathrm{mg} / \mathrm{L})$ & 2,06 & $1,0 \times 10^{-4}$ \\
$\mathrm{Ca}^{2+}(\mathrm{mg} / \mathrm{L})$ & 3,68 & $1,0 \times 10^{-5}$ \\
$\mathrm{Mg}^{2+}(\mathrm{mg} / \mathrm{L})$ & 1,12 & $1,0 \times 10^{-6}$ \\
$\mathrm{Cl}^{-}(\mathrm{mg} / \mathrm{L})$ & 11,1 & $1,0 \times 10^{-7}$ \\
$\mathrm{HCO}_{3-}(\mathrm{mg} / \mathrm{L})$ & 6,03 & $1,0 \times 10^{-8}$ \\
$\mathrm{SO}_{4}{ }^{2-}(\mathrm{mg} / \mathrm{L})$ & 0,02 & $1,0 \times 10^{-9}$ \\
$\mathrm{NO}_{3}^{-}(\mathrm{mg} / \mathrm{L})$ & 48 & $1,0 \times 10^{-10}$ \\
$\mathrm{~F}^{-}(\mathrm{mg} / \mathrm{L})$ & 0,14 & 0 \\
$\mathrm{Etanol}^{(\mathrm{g} / \mathrm{L})}$ & $1,0 \times 10^{-30}$ & 50 \\
\hline \hline
\end{tabular}

\section{RESULTADOS}

As simulações permitiram avaliar as variações temporais nas concentrações do $\mathrm{NO}_{3}$ e etanol, que serviram como referência para avaliação da eficiência da remediação. A partir dos pontos de injeção foi criado um gradiente de potencial hidráulico que irá abranger parte significativa do modelo (Figura 4a). Em função deste gradiente de pressão, a pluma se etanol se espalhou radialmente a partir dos pontos de injeção (Figura 4b).
A partir de sua posição inicial, a pluma de etanol é transportada para jusante e sua biodegradação via redução do nitrato passa a promover fortes decréscimos nas concentrações de nitrato na água subterrânea. A evolução da pluma de nitrato ao longo de 900 dias de simulação é apresentada na Figura 5. 
Figura 4 - a) Distribuição de potencial hidráulico durante a injeção; b) Concentração de etanol imediatamente após o término da injeção
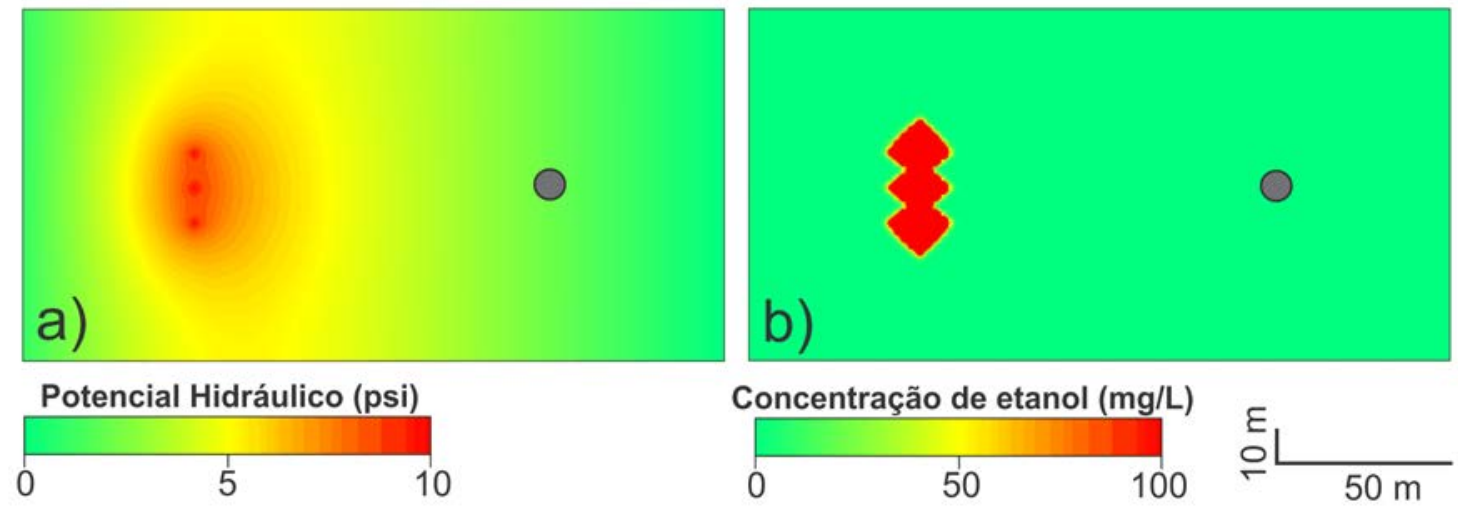

Poço de bombeamento

Figura 5 - Concentrações simuladas de nitrato: a) 100 dias; b) 300 dias; c) 500 dias; d) 700 dias; e) 900 dias.
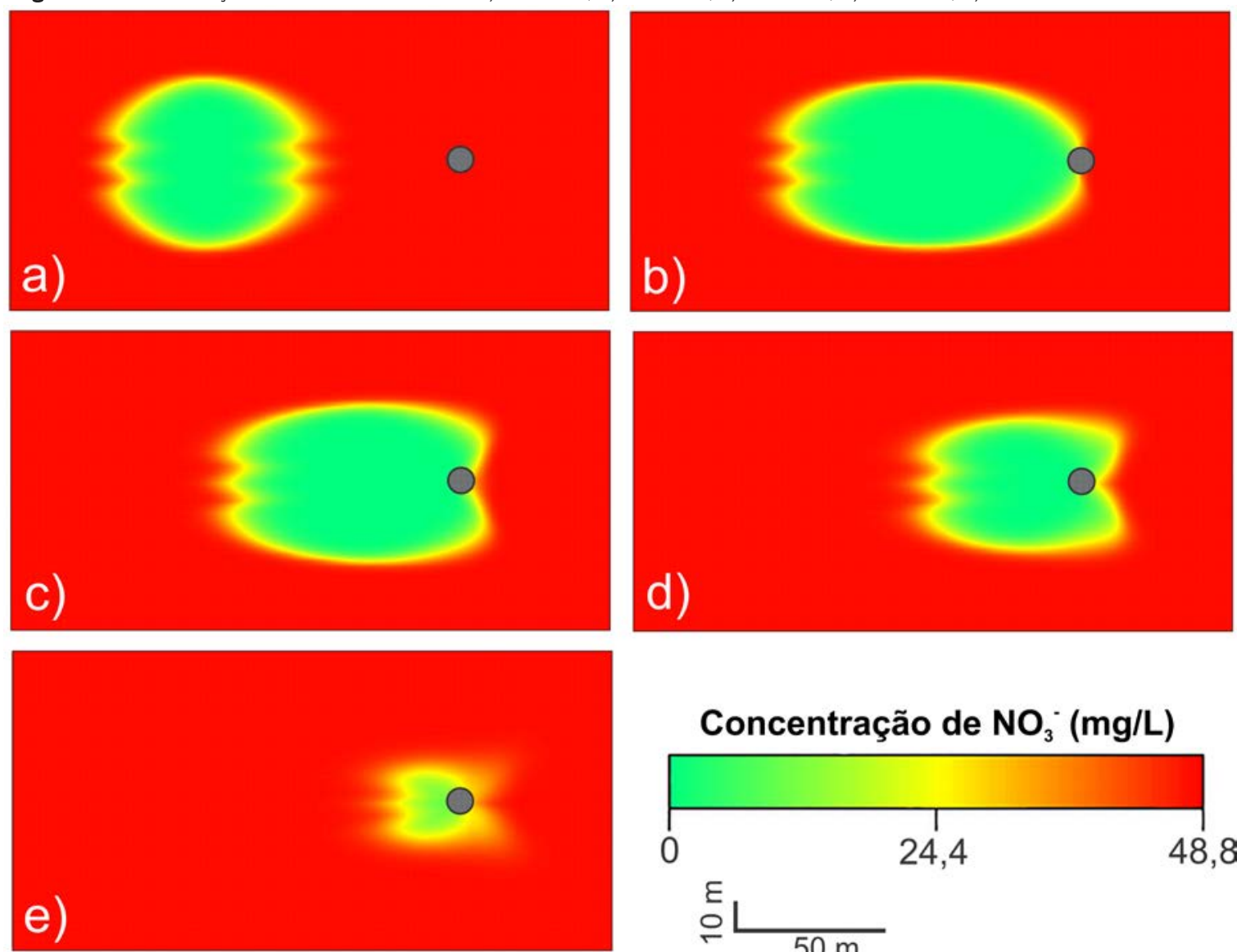

\section{Concentração de $\mathrm{NO}_{3}^{-}(\mathrm{mg} / \mathrm{L})$}

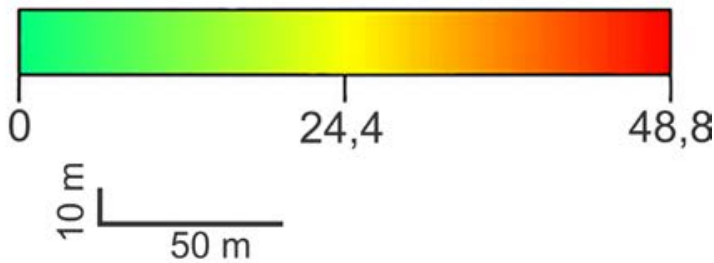

\section{Poço de bombeamento}

A Figura 6 apresenta a concentração de nitrato no poço de bombeamento ao longo dos 900 dias de simulação. A água remediada em relação ao nitrato, leva em torno de 230 dias para chegar ao poço de bombeamento e, a partir deste ponto, o poço de bombeamento irá extrair água com concentração de nitrato abaixo de 44 $\mathrm{mg} / \mathrm{L}$, isto é, dentro dos limites de potabilidade, nos 470 dias subsequentes de simulação. A concentração mínima observada no

poço de bombeamento foi de $4,98 \mathrm{mg} / \mathrm{L}$ no $620^{\circ}$ dia de simulação. Entretanto, como é possível observar na Figura 6, a continuidade da extração de água subterrânea e o consumo do etanol injetado irá promover a elevação na concentração de nitrato no poço de bombeamento a partir de 700 dias, caso não haja a eliminação da fonte de contaminação (vazamentos da rede de esgoto ou fossas sépticas). 
Figura 6 - Gráfico de evolução das concentrações de $\mathrm{NO}_{3}{ }^{-}$no poço de bombeamento

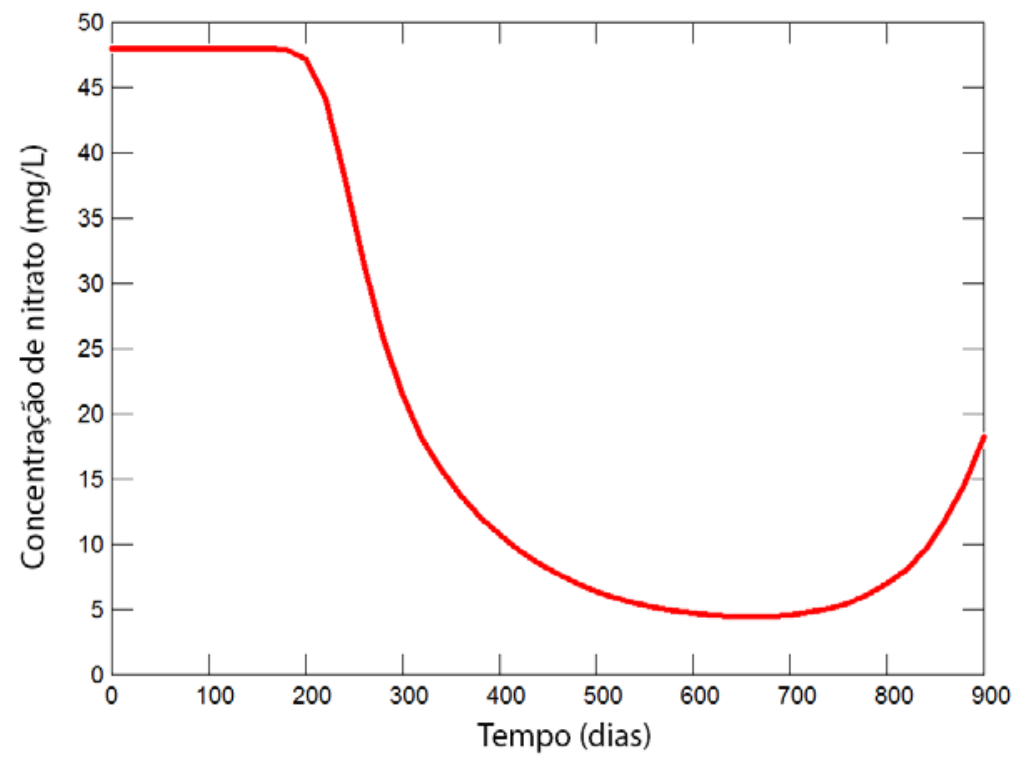

\section{DISCUSSÕES}

O nitrato é o principal contaminante da água subterrânea, mas poucas pesquisas têm sido desenvolvidas para o aprimoramento de técnicas baseadas em seu tratamento in situ. Buscando avaliar a eficiência da remediação do nitrato a partir da injeção de etanol em escala de campo e as condições operacionais necessárias para o seu sucesso, foram utilizadas simulações de transporte reativo, ferramentas que permitem a previsão da eficiência, bem como a otimização das condições operacionais, tais como pressão de injeção, concentração de solução e distância dos pontos de injeção, possibilitando a minimização do erro.

Os resultados da simulação demonstram que injeção de pulsos de solução contendo etanol são suficientes para descontaminação do aquífero a jusante do poço de bombeamento, permitindo a extração de água com concentrações de nitrato abaixo do limite de potabilidade por um período superior a 1 ano. Desta maneira, a injeção periódica de etanol pode promover a descontaminação do nitrato da água subterrânea a montante do poço de bombeamento, permitindo a extração de água apropriada para o consumo humano.

Para assegurar a eficiência do sistema de remediação testado, contudo, é imperativo que a injeção ocorra ao longo das linhas de fluxo que convergem para os poços de bombeamento. De maneira similar, ressalta-se a necessidade de se efetuar testes de bombeamento para determinar a zona de captura dos poços de bombeamento, a fim de se planejar o número de pontos de injeção e o espaçamento entre estes pontos.

O sucesso do sistema testado está relacionado às características do aquífero, uma vez que a distância que a solução contendo etanol migrará durante a injeção é função da condutividade hidráulica do aquífero. Deste modo, em aquíferos pouco permeáveis ou com abundância de lentes argilosas, a eficiência deste método será limitada. Tendo em vista que as condições reproduzidas neste modelo são de um aquífero homogêneo e permeável, é necessário avaliar a influência de heterogeneidades litológicas na injeção da solução contendo etanol.
O trabalho de Zhang et al. (2006) identificou um tempo de meiavida do etanol em torno de 2,2 dias. Diante da elevada reatividade do etanol, o sucesso da remediação está relacionado à distância em que a solução contendo etanol irá alcançar durante a injeção. Quando todo o nitrato é consumido na biodegradação do etanol, o etanol remanescente será rapidamente biodegradado por metanogênese e não estará disponível para biodegradação do nitrato a longo prazo.

Diante da eficiência do etanol na redução do nitrato e de seu baixo custo relativo, as técnicas de remediação baseadas em sua injeção são viáveis e devem ser avaliadas em testes de campo, com monitoramento prolongado, para validar as simulações realizadas e identificar possíveis limitações de sua representatividade.

O tempo requerido para a descontaminação do aquífero após a eliminação das fontes de contaminação pode abranger períodos de anos ou décadas, dependendo das dimensões das plumas de nitrato e das características do aquífero contaminado. Em razão deste contexto, salienta-se que o tipo de remediação proposto neste trabalho deve ser empregado apenas como uma medida para acelerar a descontaminação do aquífero, enquanto a eliminação das fontes de contaminação, tais como fossas sépticas e vazamentos da rede de esgoto, representam as ações mais importantes e urgentes. A não eliminação das fontes de contaminação promoverá o reaparecimento de elevadas concentrações de nitrato nos poços de abastecimento.

\section{CONCLUSÕES}

Os testes conduzidos nas simulações realizadas permitiram verificar que a remediação por pulsos individuais de injeção de etanol pode representar um método eficiente para remediação de água subterrânea contaminada por nitrato. A implantação da remediação do nitrato com a injeção de etanol em 3 pontos situado 150 metros a montante do poço de abastecimento resultará na descontaminação de um volume significativo de água que propiciar a extração de água apropriada para consumo por 470 dias. Em períodos posteriores, é observada a ressurgiria da contaminação 
em razão do transporte de água subterrânea a jusante da área remediada pela injeção de etanol. Deste modo, fica evidente que esta técnica é viável a longo prazo apenas se for acompanhada de trabalhos que promovam a eliminação das fontes ativas de contaminação. Os resultados deste trabalho indicam que este modelo apresentou-se viável com bons resultados, mas trabalhos em escala de campo, ainda escassos, devem ser conduzidos para aprimorar o seu entendimento e possível aplicação prática no futuro.

\section{REFERÊNCIAS}

BETHKE, C.M.; YEAKEL, S. The Geochemist's Workbench Reference Manual, Release 11.0. Aqueous Solutions, LLC. 2014.

CASTRO, Sérgio C.S. et al. Contaminação por água subterrânea em São José do Rio Preto (SP): contaminação por nitratos. Águas Subterrâneas, 1992.

EXNER, Mary E.; HIRSH, Aaron J.; SPALDING, Roy F. Nebraska's groundwater legacy: Nitrate contamination beneath irrigated cropland. Water resources research, v. 50, n. 5, p. 4474-4489, 2014.

FU, F.; DIONYSIOU, D. D.; LIU, H. The use of zero-valent iron for groundwater remediation and wastewater treatment: a review. Journal of hazardous materials, v. 267, p. 194-205, 2014.

GOMEZ, M. A.; GONZÁLEZ-LÓPEZ, J.; HONTORIA-GARCIA, E. Influence of carbon source on nitrate removal of contaminated groundwater in a denitrifying submerged filter. Journal of hazardous materials, v. 80, n. 1-3, p. 6980, 2000.

GONÇALVES, R. D.; CHANG, K.H. Condutividade Hidráulica da Formação Rio Claro a partir de Ensaios granulométricos. Holos Environment, v. 18 , n. 1 , p. $44-58.2018$

GRIMMEISEN, F. et al. Isotopic constraints on water source mixing, network leakage and contamination in an urban groundwater system. Science of the Total Environment, v. 583, p. 202-213, 2017.

HUNTER, W. J.; FOLLETT, R. F.; CARY, J. W. Use of vegetable oil to remove nitrate from flowing groundwater. Transactions of the ASAE, v. 40, n. 2, p. 345-353, 1997.

LOCKHART, K. M.; KING, A. M.; HARTER, T. Identifying sources of groundwater nitrate contamination in a large alluvial groundwater basin with highly diversified intensive agricultural production. Journal of contaminant hydrology, v. 151, p. 140-154, 2013.

MCMAHON, P.B.; DENNEHY, K. F.; BRUCE, B.W. In-situ Bioremediation of Nitrate-contaminated Ground Water: A Pilot Test, Julesburg, Colorado, 1996-97. US Department of the Interior, US Geological Survey, 1998.

MARTIN, Derek et al. Acetate and ethanol as potential enhancers of low temperature denitrification in soil contaminated by fur farms: a pilot-scale study. Journal of hazardous materials, v. 163, n. 2-3, p. 1230-1238, 2009.

MELO, J. G.; QUEIROZ, M. A.; HUNZIKER, J. Mecanismos e fontes de contaminação das águas subterrâneas de Natal/RN por nitrato. Águas Subterrâneas, n. 1, 1998.

MOHSENI-BANDPI, A.; ELLIOTT, D. J. Groundwater denitrification with alternative carbon sources. Water Science and Technology, v. 38, n. 6, p. 237-243, 1998.

MONTANHEIRO, F. Contaminação por nitrato no Aquífero Adamantina: o caso do município de Monte Azul Paulista - SP. Dissertação (Mestrado)-
Instituto de Geociências e Ciências Exatas, Universidade Estadual Paulista Júlio de Mesquita Filho, Rio Claro, 177 f. 2014.

NETO, D.C.; CHANG, Hung K.; VAN GENUCHTEN, M.T. A mathematical view of water table fluctuations in a shallow aquifer in Brazil. Groundwater, v. 54, n. 1, p. 82-91, 2016

OLIVA, A.; CHANG, K.H.; CAETANO-CHANG, M.R. Determinação da condutividade hidráulica da Formação Rio Claro: análise comparativa através de análise granulométrica e ensaios com permeâmetro guelph e testes de slug. Águas subterrâneas, v. 19, n. 2, 2005.

OLIVA, A. Estudo hidrofaciológico do aquífero Rio Claro no município de Rio Claro-SP. 244 f. 2006.

PU, J, et al. Pyrite-based autotrophic denitrification for remediation of nitrate contaminated groundwater. Bioresource Technology, v. 173, p. 117 123, 2014.

REYNOLDS, J. H.; BARRETT, M.H. A review of the effects of sewer leakage on groundwater quality. Water and Environment Journal, v. 17, n. 1, p. 34 39, 2003.

ROBERTSON, W. D. et al. Long-term performance of in situ reactive barriers for nitrate remediation. Groundwater, v. 38, n. 5, p. 689-695, 2000.

SALMINEN, Jani M. et al. Ethanol-based in situ bioremediation of acidified nitrate-contaminated groundwater. Water research, v. 63, p. 306-315, 2014.

SCHIPPER, L.A.; VOJVODIĆ-VUKOVIĆ, M. Nitrate removal from groundwater and denitrification rates in a porous treatment wall amended with sawdust. Ecological Engineering, v. 14, n. 3, p. 269-278, 2000.

SMITH, R. L. et al. In situ stimulation of groundwater denitrification with formate to remediate nitrate contamination. Environmental science \& technology, v. 35, n. 1, p. 196-203, 2001.

SU, C.; PULS, R. W. Removal of added nitrate in cotton burr compost, mulch compost, and peat: mechanisms and potential use for groundwater nitrate remediation. Chemosphere, v. 66, n. 1, p. 91-98, 2007.

TAI, Y.; DEMPSEY, B. A. Nitrite reduction with hydrous ferric oxide and Fe (II): stoichiometry, rate, and mechanism. Water research, v. 43, n. 2, p. 546-552, 2009.

TORRENTÓ, Clara et al. Enhanced denitrification in groundwater and sediments from a nitrate-contaminated aquifer after addition of pyrite. Chemical Geology, v. 287, n. 1-2, p. 90-101, 2011.

VYSTAVNA, Y. et al. Nitrate contamination in a shallow urban aquifer in East Ukraine: evidence from hydrochemical, stable isotopes of nitrate and land use analysis. Environmental Earth Sciences, v. 76, n. 13, p. 463, 2017.

WANG, L. et al. The changing trend in nitrate concentrations in major aquifers due to historical nitrate loading from agricultural land across England and Wales from 1925 to 2150. Science of the Total Environment, v. 542, p. 694-705, 2016

WEITZBERG, Eddie; LUNDBERG, Jon O. Novel aspects of dietary nitrate and human health. Annual review of nutrition, v. 33, p. 129-159, 2013.

ZHANG, Y.; KHAN, I. A.; CHEN, X. H.; SPALDING, R. F. Transport and degradation of ethanol in groundwater. Journal of Contaminant Hydrology, v. 82, n. 3-4, p. 183-194, 2006.

ZHANG, Qianqian et al. Driving mechanism and sources of groundwater nitrate contamination in the rapidly urbanized region of south China. Journal of Contaminant Hydrology, v. 182, p. 221-230, 2015. 